\title{
Editorial: Peer-to-peer networking and applications, volume 2 , issue 4
}

\author{
Xuemin Shen • Heather Yu
}

Published online: 27 August 2009

(C) Springer Science + Business Media, LLC 2009

Six original research works are included in this issue of the Peer-to-Peer Networking and Applications. They address research problems related to resource discovery, information dissemination and supporting complex queries in computational Grid, command and control for distributed multi-agent systems, network partitioning, and peer-to-peer networks supporting business activities.

Grid computing has received significant attention from researchers and application developers. In a Grid, computing resources are distributed over the willing participants with continually varying resources. Thus, resource discovery is one the most crucial components to determine the efficiency of the entire system. In the first paper of this issue, "Facilitating Resource Discovery in Grid Environments with Peer-to-Peer Structured Tuple Spaces", Li et al. propose RDSpace, a substrate to enable efficient resource discovery in a grid environment. Unlike the traditional tree structured centralized resource discovery service entities, RDSpace resource information is organized in a peer-topeer (P2P) structured tuple space. It is demonstrated that RDSpace is scalable, flexible and fault-tolerant.

In the second paper, "Gossiping Protocols for Hybrid Grid Resource Scheduling", Erdil and Lewis address the problem of information dissemination in Grid. They investigate performance characteristics of three information

\footnotetext{
X. Shen $(\triangle)$

Department of ECE, University of Waterloo,

Waterloo, Ontario, Canada

e-mail: xshen@bbcr.uwaterloo.ca

H. Yu

Huawei Technologies,

Bridgewater, NJ, USA

e-mail: heathery@ieee.org
}

dissemination techniques - pair wise gossiping, epidemic gossiping and adaptive gossiping, and report the findings.

Though the concept of P2P networks started its journey to facilitate file sharing, many other applications have been evolved to exploit different interesting features of these highly scalable and robust networks. In the third paper, "Structured P2P Technologies for Distributed Command and Control", Karrels et al. evaluate existing P2P architectures to suitably deploy a robust command and control system for distributed multi-agent systems.

Structured overlays, particularly ring based overlays, create a significant portion of current P2P systems. Many of these overlays are shown to be scalable, fault-tolerant and selfmanaging. Though the problem of peer churn and network partitioning of ring-based overlays have received significant attention from the research community, the problem of network merging has not been studied. In the forth paper, "Dealing with Network Partitions in Structured Overlay Networks", Shafaat et al. propose an algorithm to merge multiple similar ringbased overlays when the underlying network merges.

The fifth paper of this issue addresses the problem of complex queries in Grid networks. Queries for resources are typically composed of multiple attributes with ranges, and traditional scalable P2P solutions do not support these complex queries. Other solutions support rich queries but are not reliable or scalable. In "NodeWiz: Fault-Tolerant Grid Information Service", Basu et al. propose NodeWiz which supports efficient multi-attribute range queries, and is reliable and load balanced.

A digital ecosystem for business is a digital infrastructure that enables business organizations to combine services to perform core business activities or to offer integrated solutions. In the last paper of this issue, "An Open Digital Environment to Support Business Ecosystems", Razavi et al. propose a completely distributed P2P network that 
supports long-running transactions between the networked business organizations. They focus on the network structures formed by interrelationship between the local interactions, and demonstrate a way to establish a P2P network of interconnected smaller networks.

In closing, we would like to acknowledge the contributions of the authors who submitted their works and the reviewers who provided their expert opinions and constructive and helpful comments. We would also like to extend our sincere thanks to Melissa Fearon, the Senior Editor, Emma Kalb, and Cristina Chua of Springer, for their support and help in bringing forward Volume 2, Issue 4 of this journal. We hope that you will enjoy the current issue and find the journal useful.

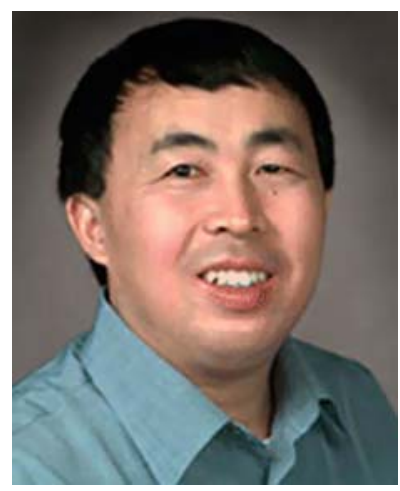

Xuemin (Sherman) Shen (IEEE M'97-SM'02-F'09) received the B.Sc. (1982) degree from Dalian Maritime University (China) and the M.Sc. (1987) and Ph.D. degrees (1990) from Rutgers University, New Jersey (USA), all in electrical engineering. $\mathrm{He}$ is a Professor and University Research Chair, Department of Electrical and Computer Engineering, University of Waterloo, Canada. Dr. Shen's research focuses on mobility and resource management in interconnected wireless/wired networks, UWB wireless communications networks, wireless network security, wireless body area networks and vehicular ad hoc and sensor networks. He is a co-author of three books, and has published more than 400 papers and book chapters in wireless communications and networks, control and filtering. Dr. Shen served as the Tutorial Chair for IEEE ICC'08, the Technical Program Committee Chair for IEEE Globecom'07, the General Co-Chair for Chinacom'07 and QShine'06, the Founding Chair for IEEE Communications Society Technical Committee on P2P Communications and Networking. He also serves as a Founding Area Editor for IEEE Transactions on Wireless
Communications; Editor-in-Chief for Peer-to-Peer Networking and Application; Associate Editor for IEEE Transactions on Vehicular Technology; KICS/IEEE Journal of Communications and Networks, Computer Networks; ACM/Wireless Networks; and Wireless Communications and Mobile Computing (Wiley), etc. He has also served as Guest Editor for IEEE JSAC, IEEE Wireless Communications, IEEE Communications Magazine, and ACM Mobile Networks and Applications, etc. Dr. Shen received the Excellent Graduate Supervision Award in 2006, and the Outstanding Performance Award in 2004 and 2008 from the University of Waterloo, the Premier's Research Excellence Award (PREA) in 2003 from the Province of Ontario, Canada, and the Distinguished Performance Award in 2002 and 2007 from the Faculty of Engineering, University of Waterloo. Dr. Shen is a registered Professional Engineer of Ontario, Canada, and a Distinguished Lecturer of IEEE Communications Society.

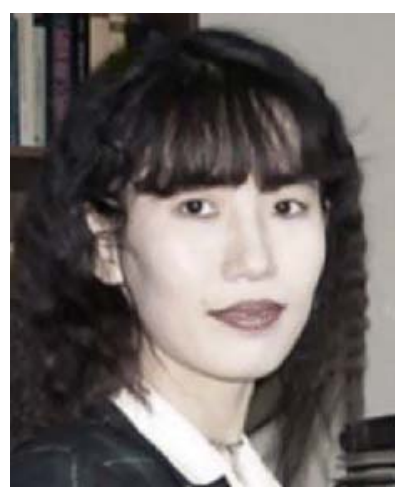

Heather Yu received her Ph.D. in Electrical Engineering from Princeton University in 1998. From September 1998 to March 2007, she was with Panasonic Princeton Laboratory where her major focus was multimedia communication and multimedia security R\&D. Currently she is the Senior Manager of Media Technologies at Huawei Technologies USA. One of her responsibilities is to lead the establishment of the multimedia content networking research team in New Jersey,

USA. Her current research interests include human centric multimedia communications, IPTV, Peer-to-peer networking, mobile multimedia, etc. She is serving as the Associate Editor-in-Chief of the Journal of Peer-to-Peer Networking and Applications, Associate Editor of IEEE Multimedia Magazine and IEEE Communications Surveys and Tutorials, and Chair of the Human Centric Technical Sub-committee of IEEE ComSoc. She served as Technical Program Chair for several IEEE multimedia and communications conferences, the Chair of IEEE Multimedia Communications Technical Committee, and a voting member at ComSoc GITC, ComSoc SPC(strategic planning committee), etc. She has published 2 books, more than 60 technical papers and holds 23 US patents. 